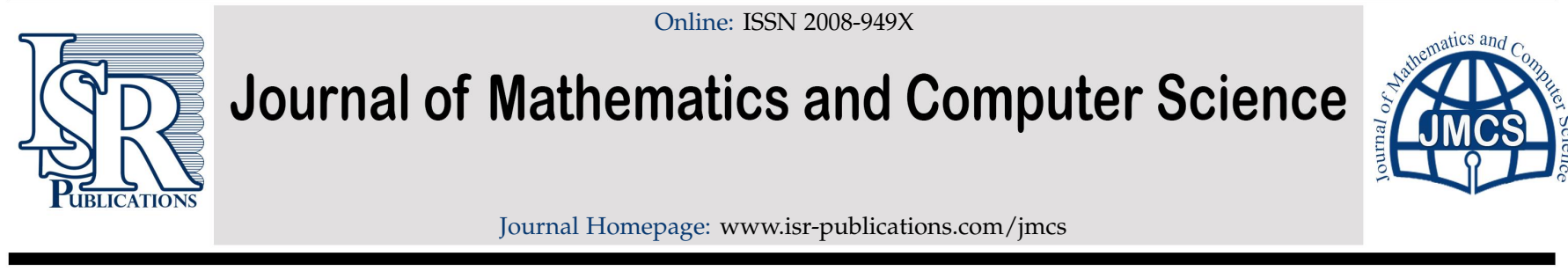

\title{
On BF-semigroups and Fuzzy BF-semigroups
}

Mae Manahon ${ }^{\mathrm{a}}$, Jenette Bantug ${ }^{\mathrm{b}}$, Joemar Endam ${ }^{\mathrm{a}, *}$

a Mathematics Department, Negros Oriental State University, Dumaguete City, Philippines.

${ }^{b}$ Mathematics Department, Silliman University, Dumaguete City, Philippines.

\begin{abstract}
We introduce and establish the notion of BF-semigroups. We construct quotient BF-semigroups via BF-ideals and we investigate homomorphisms of BF-semigroups and establish the isomorphism theorems for BF-semigroups. Moreover, we apply the concept of fuzzy sets to BF-semigroups.
\end{abstract}

Keywords: $\mathrm{BF} / \mathrm{BF}_{1} / \mathrm{BF}_{2}$-semigroup, sub BF-semigroup, $\mathrm{BF}$-ideal, quotient BF-semigroup, fuzzy sub BF-semigroup. 2010 MSC: 06F35, 08A05, 03G25, 94D05.

(C)2020 All rights reserved.

\section{Introduction}

The concept of $\mathrm{BH}$-algebras was introduced by Jun et al. [4]. They defined a BH-algebra as an algebra of $(A ; *, 0)$ of type $(2,0)$ (that is, a nonempty set $A$ with a binary operation $*$ and a constant 0$)$ satisfying the following axioms:

(B1) $x * x=0$;

(B2) $x * 0=x$;

(BH) $x * y=0$ and $y * x=0$ imply $x=y$.

In [5], Kim and Kim introduced BG-algebras. An algebra $(A ; *, 0)$ of type $(2,0)$ is a BG-algebra if it obeys (B1), (B2), and

(BG) $x=(x * y) *(0 * y)$.

In [7], Walendziak introduced $B F$-algebras, together with $\mathrm{BF}_{1} / \mathrm{BF}_{2}$-algebras. An algebra $(A ; *, 0)$ of type $(2,0)$ is a BF-algebra if it obeys (B1), (B2), and

\footnotetext{
*Corresponding author

Email addresses: maedmanahon513@gmail.com (Mae Manahon), jenettesbantug@su.edu.ph (Jenette Bantug), joemar.endam@norsu.edu.ph (Joemar Endam)
}

doi: $10.22436 /$ jmcs.020.04.06

Received: 2019-07-16 Revised: 2020-02-06 Accepted: 2020-02-10 
(BF) $0 *(x * y)=y * x$.

$A B_{1}$-algebra is a $B F$-algebra satisfying $(B G)$ and a $B F_{2}$-algebra is a $B F$-algebra satisfying $(B H)$. Let $(A ; *, 0)$ be a BF-algebra. A nonempty subset $N$ of $A$ is called a subalgebra of $A$ if $x * y \in N$ for any $x, y \in N$. $A$ subset $I$ of $A$ is called an ideal of $A$ if it satisfies:

(I1) $0 \in \mathrm{I}$;

(I2) $x * y \in I$ and $y \in$ I imply $x \in I$.

We say that an ideal I is normal if it satisfies:

(I3) $x * y \in \mathrm{I}$ implies $(z * x) *(z * y) \in \mathrm{I}$, for any $z \in A$.

The concept of fuzzy set, which was introduced by Zadeh [8] provides an extension of the classical notion of set. Since then a number of researches, both in theory and application, involving fuzzy sets have been established. In particular, Borumand Saeid and Rezvani [1] introduced fuzzy BF-algebras and they established some properties of this concept. Moreover, Hadipour [3] generalized the concept of fuzzy BFalgebras. We introduce and establish the notion of BF-semigroups. We construct quotient BF-semigroups via BF-ideals and we investigate homomorphisms of BF-semigroups and establish the isomorphism theorems for BF-semigroups. Moreover, we apply the concept of fuzzy sets to BF-semigroups.

\section{BF-semigroups}

Definition 2.1. A BF-semigroup is a nonempty set $X$ together with two binary operations $*$ and $\cdot$ and a constant 0 satisfying:

(i) $(\mathrm{X} ; *, 0)$ is a BF-algebra;

(ii) $(X, \cdot)$ is a semigroup;

(iii) $x \cdot(y * z)=(x \cdot y) *(x \cdot z)$ and $(x * y) \cdot z=(x \cdot z) *(y \cdot z)$.

Definition 2.2. A BF-semigroup is called a $\mathrm{BF}_{1}$-semigroup (resp. a $\mathrm{BF}_{2}$-semigroup) if it obeys (BG) (resp. $(\mathrm{BH})$ ).

Example 2.3. Let $X=\{0, a, b, c\}$ be a set with the following table of operations:

\begin{tabular}{c|ccccc|cccc}
$*$ & 0 & $\mathrm{a}$ & $\mathrm{b}$ & $\mathrm{c}$ & $\cdot$ & 0 & $\mathrm{a}$ & $\mathrm{b}$ & $\mathrm{c}$ \\
\hline 0 & 0 & $\mathrm{c}$ & $\mathrm{b}$ & $\mathrm{a}$ & 0 & 0 & 0 & 0 & 0 \\
$\mathrm{a}$ & $\mathrm{a}$ & 0 & $\mathrm{c}$ & $\mathrm{b}$ & $\mathrm{a}$ & 0 & $\mathrm{~b}$ & 0 & $\mathrm{~b}$ \\
$\mathrm{~b}$ & $\mathrm{~b}$ & $\mathrm{a}$ & 0 & $\mathrm{c}$ & $\mathrm{b}$ & 0 & 0 & 0 & 0 \\
$\mathrm{c}$ & $\mathrm{c}$ & $\mathrm{b}$ & $\mathrm{a}$ & 0 & $\mathrm{c}$ & 0 & $\mathrm{~b}$ & 0 & $\mathrm{~b}$
\end{tabular}

Then $(X ; *, \cdot, 0)$ is a BF-semigroup.

Example 2.4. Let $X=\{0, a, b\}$ be a set with the following table of operations:

\begin{tabular}{l|llll|lll}
$*$ & 0 & $\mathrm{a}$ & $\mathrm{b}$ & $\cdot$ & 0 & $\mathrm{a}$ & $\mathrm{b}$ \\
\hline 0 & 0 & $\mathrm{a}$ & $\mathrm{b}$ & 0 & 0 & 0 & 0 \\
$\mathrm{a}$ & $\mathrm{a}$ & 0 & $\mathrm{a}$ & $\mathrm{a}$ & 0 & $\mathrm{~b}$ & 0 \\
$\mathrm{~b}$ & $\mathrm{~b}$ & $\mathrm{a}$ & 0 & $\mathrm{~b}$ & 0 & 0 & 0
\end{tabular}

Then $(X ; *, \cdot, 0)$ is a BF-semigroup.

Example 2.5. Let $X=\{0, a, b\}$ be a set with the following table of operations: 


\begin{tabular}{l|llll|lll}
$*$ & 0 & $\mathrm{a}$ & $\mathrm{b}$ & $\cdot$ & 0 & $\mathrm{a}$ & $\mathrm{b}$ \\
\hline 0 & 0 & $\mathrm{a}$ & $\mathrm{b}$ & 0 & 0 & 0 & 0 \\
$\mathrm{a}$ & $\mathrm{a}$ & 0 & 0 & $\mathrm{a}$ & 0 & $\mathrm{a}$ & $\mathrm{b}$ \\
$\mathrm{b}$ & $\mathrm{b}$ & 0 & 0 & $\mathrm{~b}$ & 0 & $\mathrm{~b}$ & $\mathrm{a}$
\end{tabular}

Then $(\mathrm{X} ; *, \cdot, 0)$ is a BF-semigroup.

Example 2.6. If $(X ; *, 0)$ is a BF-algebra, then $(X ; *, \cdot, 0)$ is a BF-semigroup, where $x \cdot y=0$ for any $x, y \in X$.

Every $\mathrm{BF}_{1}$-semigroup is a $\mathrm{BF}_{2}$-semigroup (see [7, Proposition 2.12]). The BF-semigroup in Example 2.5 is not a $B F_{2}$-semigroup since $a * b=0$ and $b * a=0$ but $a \neq b$. Example 2.4 is a $B_{2}$-semigroup which is not a $\mathrm{BF}_{1}$-semigroup since $(\mathrm{b} * \mathrm{a}) *(0 * \mathrm{a})=\mathrm{a} * \mathrm{a}=0 \neq \mathrm{b}$.

Proposition 2.7 ([7, Proposition 2.5]). If $(A ; *, 0)$ is a BF-algebra, then for any $x, y \in A$,

(a) $0 *(0 * x)=x$;

(b) $0 * x=0 * y$ implies $x=y$;

(c) $x * y=0$ implies $y * x=0$.

From now on, let $\mathrm{X}$ stands for BF-semigroup $(X ; *, \cdot, 0)$.

Proposition 2.8. For all $a, b, c \in X$,

(a) $a \cdot 0=0 \cdot a=0 ;$

(b) $a \cdot(0 * b)=(0 * a) \cdot b=0 *(a \cdot b)$;

(c) $(0 * a) \cdot(0 * b)=a \cdot b$;

(d) $a \cdot(b *(0 * c))=(a \cdot b) *(0 *(a \cdot c)),(b *(0 * c)) \cdot a=(b \cdot a) *(0 *(c \cdot a))$.

Proof.

(a): By (B2), distributive property, and (B1), $a \cdot 0=a \cdot(0 * 0)=(a \cdot 0) *(a \cdot 0)=0$. Similarly, $0 \cdot a=0$.

(b): By distributive property and $(a), a \cdot(0 * b)=(a \cdot 0) *(a \cdot b)=0 *(a \cdot b)=(0 \cdot b) *(a \cdot b)=(0 * a) \cdot b$.

(c): By (b), distributive property, (a), and Proposition $2.7(a),(0 * a) \cdot(0 * b)=0 *(a \cdot(0 * b))=0 *((a \cdot 0) *$ $(a \cdot b))=0 *(0 *(a \cdot b))=a \cdot b$.

(d): By distributive property and $(b), a \cdot(b *(0 * c))=(a \cdot b) *(a \cdot(0 * c))=(a \cdot b) *(0 *(a \cdot c))$. Similarly, $(b *(0 * c)) \cdot a=(b \cdot a) *(0 *(c \cdot a))$.

Definition 2.9. A nonempty subset $S$ of $X$ is called a sub BF-semigroup of $X$ if $x * y, x \cdot y \in S$ for all $x, y \in S$.

Clearly, $\{0\}$ and $X$ are sub BF-semigroups of $X$.

Example 2.10. Let $X=\{0, a, b, c\}$ be a set with the following table of operations:

\begin{tabular}{l|llll}
$*$ & 0 & $\mathrm{a}$ & $\mathrm{b}$ & $\mathrm{c}$ \\
\hline 0 & 0 & $\mathrm{a}$ & $\mathrm{b}$ & $\mathrm{c}$ \\
$\mathrm{a}$ & $\mathrm{a}$ & 0 & $\mathrm{c}$ & $\mathrm{b}$ \\
$\mathrm{b}$ & $\mathrm{b}$ & $\mathrm{c}$ & 0 & $\mathrm{a}$ \\
$\mathrm{c}$ & $\mathrm{c}$ & $\mathrm{b}$ & $\mathrm{a}$ & 0
\end{tabular}

\begin{tabular}{l|llll}
$\cdot$ & 0 & $\mathrm{a}$ & $\mathrm{b}$ & $\mathrm{c}$ \\
\hline 0 & 0 & 0 & 0 & 0 \\
$\mathrm{a}$ & 0 & $\mathrm{a}$ & $\mathrm{b}$ & $\mathrm{c}$ \\
$\mathrm{b}$ & 0 & $\mathrm{~b}$ & $\mathrm{c}$ & $\mathrm{a}$ \\
$\mathrm{c}$ & 0 & $\mathrm{c}$ & $\mathrm{a}$ & $\mathrm{b}$
\end{tabular}

Then $(X ; *, \cdot, 0)$ is a BF-semigroup. The set $S_{1}=\{0, a\}$ is a sub BF-semigroup of $X$, while the set $S_{2}=\{0, a, b\}$ is not since $\mathrm{a} * \mathrm{~b}=\mathrm{c} \notin \mathrm{S}_{2}$. 


\section{Ideals in BF-semigroups}

Definition 3.1. A nonempty subset I of $X$ is called a BF-ideal of $X$ if it obeys (I1), (I2), (I3), and

(I4) $a \cdot x, x \cdot a \in I$ for any $a \in I, x \in X$.

This means $I$ is a BF-ideal of $X$ if and only if $I$ is a normal ideal of $X$ satisfying (I4). Also, if I is a BF-ideal of $X$, then $I$ is a sub BF-semigroup of $X$. The set $I_{1}=\{0, b\}$ in Example 2.4 is a BF-ideal of $X$ while $I_{2}=\{0, a\}$ is not since $a \cdot a=b \notin I_{2}$. Clearly, $X$ is a BF-ideal of $X$. However, $\{0\}$ need not be a BF-ideal of $X$ (see [7, Example 3.2]). If $X$ is a $B_{2}$-semigroup, then $\{0\}$ is a BF-ideal of $X$. Indeed, if $x, y, z \in X$, then clearly (I1) and (I2) hold for $\{0\}$. Suppose that $x * y=0$. Then from [7, Proposition 2.9], $x=y$ and so $(z * x) *(z * y)=0$. Now, (I4) follows from Proposition 2.8(a). Therefore, $\{0\}$ is a BF-ideal of $X$.

\section{Theorem 3.2.}

(a) The intersection of sub BF-semigroups of $\mathrm{X}$ is a sub BF-semigroup of $\mathrm{X}$; and

(b) the intersection of BF-ideals of $\mathrm{X}$ is a BF-ideal of $\mathrm{X}$.

In [2], if $I$ and $J$ are ideals of BF-algebra $(X ; *, 0)$, then we define the subset IJ of $X$ to be the set $\{x \in X: x=i *(0 * j)$ for some $i \in I, j \in J\}$. From [2, Lemma 3.3(iii)], if I and J are normal ideals of a BF-algebra $(X ; *, 0)$, then for any $i_{1}, i_{2} \in I, j_{1}, j_{2} \in J$, (SI) implies IJ is a subalgebra of $X$, where (SI) is given below

$$
\text { (SI) }\left(i_{1} * j_{1}\right) *\left(i_{2} * j_{2}\right)=\left(i_{1} * i_{2}\right) *\left(j_{1} * j_{2}\right) .
$$

Proposition 3.3. Let I and J be BF-ideals of a BF-semigroup X such that X satisfies (SI). Then IJ is a sub BFsemigroup of $\mathrm{X}$.

Proof. Let $x, y \in I J$. Then there exist $i_{1}, i_{2} \in I$ and $j_{1}, j_{2} \in J$ such that $x=i_{1} *\left(0 * j_{1}\right)$ and $y=i_{2} *\left(0 * j_{2}\right)$. Since $I$ is a BF-ideal of $X$ and $i_{2} \in I,\left(i_{1} *\left(0 * j_{1}\right)\right) \cdot i_{2} \in I$. Since $J$ is a BF-ideal of $X$ and $j_{2} \in J,\left(i_{1} *\left(0 * j_{1}\right)\right)$. $j_{2} \in J$. Thus, by distributive property and Proposition $2.8(b)$, we have

$$
\begin{aligned}
x \cdot y & =\left(i_{1} *\left(0 * j_{1}\right)\right) \cdot\left(i_{2} *\left(0 * j_{2}\right)\right) \\
& =\left[\left(i_{1} *\left(0 * j_{1}\right)\right) \cdot i_{2}\right] *\left[\left(i_{1} *\left(0 * j_{1}\right)\right) \cdot\left(0 * j_{2}\right)\right] \\
& =\left[\left(i_{1} *\left(0 * j_{1}\right)\right) \cdot i_{2}\right] *\left[0 *\left(\left(i_{1} *\left(0 * j_{1}\right)\right) \cdot j_{2}\right)\right] \in I J .
\end{aligned}
$$

Therefore, IJ is a sub BF-semigroup of $X$.

Definition 3.4. A map $\varphi: X \rightarrow Y$ is called a BF-semigroup homomorphism (or simply BFs-homomorphism) if $\varphi(x * y)=\varphi(x) * \varphi(y)$ and $\varphi(x \cdot y)=\varphi(x) \cdot \varphi(y)$ for any $x, y \in X$. We denote by ker $\varphi$ the subset $\{x \in X: \varphi(x)=0\}$ of $X$ (it is the kernel of the BFs-homomorphism $\varphi$ ).

Lemma 3.5. Let $\varphi: \mathrm{X} \rightarrow \mathrm{Y}$ be a BFs-homomorphism.

(a) If $\mathrm{I}$ is a sub BF-semigroup of $\mathrm{X}$, then $\varphi(\mathrm{I})$ is a sub BF-semigroup of $\mathrm{Y}$.

(b) If $\mathrm{J}$ is a sub BF-semigroup of $\mathrm{Y}$, then $\varphi^{-1}(\mathrm{~J})$ is a sub BF-semigroup of $\mathrm{X}$.

(c) If $\mathrm{I}$ is a BF-ideal of $\mathrm{X}$ and $\varphi$ is one-to-one, then $\varphi(\mathrm{I})$ is a BF-ideal of $\varphi(\mathrm{X})$.

(d) If $\mathrm{J}$ is a BF-ideal of $\mathrm{Y}$, then $\varphi^{-1}(\mathrm{~J})$ is a BF-ideal of $\mathrm{X}$.

Proof. The proof is analogous to the proof of [2, Lemma 2.6].

If $\varphi$ is a BFs-homomorphism, then $\operatorname{ker} \varphi$ need not be a BF-ideal of $X$ (see [7, Example 3.2] with identity mapping). Moreover, there is a BFs-homomorphism with kernel equal to $\{0\}$ but is not one-to-one (see [7, Example 3.13]). 
Proposition 3.6. Let $\mathrm{X}$ and $\mathrm{Y}$ be $\mathrm{BF}_{2}$-semigroups and let $\varphi: \mathrm{X} \rightarrow \mathrm{Y}$ be a BFs-homomorphism. Then

(a) $\varphi$ is one-to-one if and only if $\operatorname{ker} \varphi=\{0\}$;

(b) $\operatorname{ker} \varphi$ is a BF-ideal of $\mathrm{X}$.

Proof. This follows from [7, Proposition 3.14].

Next, we construct quotient BF-semigroups via BF-ideals. Let I be a BF-ideal of X. Then I is a normal ideal of the BF-algebra $(X ; *, 0)$. From [7], $\left(X / I ; *^{\prime}, 0 / I\right)$ is a BF-algebra (the quotient BF-algebra of $X$ modulo I), where $*^{\prime}$ is defined by $x / I *^{\prime} y / I=(x * y) / I$. Note that for any $x \in X, x / I=\{y \in X: x \sim I y\}$ is the congruence class containing $x$, where the relation $\sim_{I}$ is defined by $x \sim_{I} y$ if and only if $x * y \in I$. Now, we define.$^{\prime}$ on $X / I$ by $x / I \cdot^{\prime} y / I=(x \cdot y) / I$. The operation $\cdot^{\prime}$ is well-defined. To see this, let $x / I, x^{\prime} / I, y / I, y^{\prime} / I \in X / I$. Suppose that $x / I=x^{\prime} / I$ and $y / I=y^{\prime} / I$. Then $x * x^{\prime} \in I$ and $y * y^{\prime} \in I$. Since I is a BF-ideal of $X$ and $y * y^{\prime} \in I$, it follows that $x \cdot\left(y * y^{\prime}\right) \in I$. By distributive property, $(x \cdot y) *\left(x \cdot y^{\prime}\right)=$ $x \cdot\left(y * y^{\prime}\right) \in I$ and so $x \cdot y \sim_{I} x \cdot y^{\prime}$. Similarly, since $x * x^{\prime} \in I$, it follows that $x \cdot y^{\prime} \sim_{I} x^{\prime} \cdot y^{\prime}$. By transitive property, $x \cdot y \sim \sim_{I} x^{\prime} \cdot y^{\prime}$. Hence, $x / I \cdot \cdot^{\prime} y / I=(x \cdot y) / I=\left(x^{\prime} \cdot y^{\prime}\right) / I=x^{\prime} / I \cdot{ }^{\prime} y^{\prime} / I$. It is easy to see that $\left(\mathrm{X} / \mathrm{I} ; *^{\prime}, \cdot^{\prime}, 0 / \mathrm{I}\right)$ is a BF-semigroup. This BF-semigroup is called the quotient BF-semigroup of $\mathrm{X}$ modulo I.

Theorem 3.7. Let $\mathrm{X}$ and $\mathrm{Y}$ be $\mathrm{BF}_{2}$-semigroups and let $\varphi: \mathrm{X} \rightarrow \mathrm{Y}$ be a BFs-homomorphism from $\mathrm{X}$ onto $\mathrm{Y}$. Then $\mathrm{X} / \operatorname{ker} \varphi$ is isomorphic to $\mathrm{Y}$.

Proof. The proof is analogous to the proof of [7, Theorem 3.16].

If $I$ and $J$ are BF-ideals of $X$, then $I \cap J$ is a BF-ideal of $X$. Since $I$ is sub BF-semigroup of $X, I \cap J$ is a BF-ideal of $I$. Thus, $I /(I \cap J)$ is well-defined. If $X$ satisfies (SI), then by Proposition 3.3 and [2, Lemma 3.3 (i)], (IJ)/J is well-defined.

Theorem 3.8. If $\mathrm{I}$ and $\mathrm{J}$ are BF-ideals of $\mathrm{X}$ such that $\mathrm{X}$ satisfies (SI), then $\mathrm{I} /(\mathrm{I} \cap \mathrm{J})$ is isomorphic to $(\mathrm{IJ}) / \mathrm{J}$.

Proof. The proof is analogous to the proof of [2, Theorem 3.4].

Lemma 3.9. If $\mathrm{I}$ and $\mathrm{J}$ are BF-ideals of a BF-semigroup $\mathrm{X}$ such that $\mathrm{I} \subseteq \mathrm{J}$, then $\mathrm{J} / \mathrm{I}$ is a BF-ideal of $\mathrm{X} / \mathrm{I}$.

Proof. From [2, Lemma 3.5], $\mathrm{J} / \mathrm{I}$ is a normal ideal of $\mathrm{X} / \mathrm{I}$. Let $\mathrm{j} / \mathrm{I} \in \mathrm{J} / \mathrm{I}$ and $\mathrm{x} / \mathrm{I} \in \mathrm{X} / \mathrm{I}$. Then $\mathrm{j} \in \mathrm{J}$ and $x \in X$. Since $J$ is a BF-ideal, $j \cdot x, x \cdot j \in J$. Hence, $j / I \cdot x / I=(j \cdot x) / I \in J / I$ and $x / I \cdot j / I=(x \cdot j) / I \in J / I$. Therefore, $\mathrm{J} / \mathrm{I}$ is a BF-ideal of $\mathrm{X} / \mathrm{I}$.

Let $\mathrm{I}$ and $\mathrm{J}$ be BF-ideals of a $\mathrm{BF}_{2}$-semigroup $\mathrm{X}$ such that $\mathrm{I} \subseteq \mathrm{J}$. Then by Lemma 3.9, $(\mathrm{X} / \mathrm{I}) /(\mathrm{J} / \mathrm{I})$ is well-defined.

Theorem 3.10. If $\mathrm{I}$ and $\mathrm{J}$ are $\mathrm{BF}$-ideals of a $\mathrm{BF}_{2}$-semigroup $\mathrm{X}$ such that $\mathrm{I} \subseteq \mathrm{J}$, then $(\mathrm{X} / \mathrm{I}) /(\mathrm{J} / \mathrm{I})$ is isomorphic to $\mathrm{X} / \mathrm{J}$.

Proof. The proof is analogous to the proof of [2, Theorem 3.6].

\section{Fuzzy BF-semigroups}

Definition 4.1. A fuzzy set $\mu$ of a BF-semigroup $X$ is called a fuzzy sub BF-semigroup of $X$ if it satisfies the following axioms for all $x, y \in X$ :

(i) $\mu(x * y) \geqslant \min \{\mu(x), \mu(y)\}$;

(ii) $\mu(x \cdot y) \geqslant \min \{\mu(x), \mu(y)\}$.

Example 4.2. Let $X=\{0, a, b, c\}$ be a set with the following table of operations: 


\begin{tabular}{c|cccc}
$*$ & 0 & $\mathrm{a}$ & $\mathrm{b}$ & $\mathrm{c}$ \\
\hline 0 & 0 & $\mathrm{a}$ & $\mathrm{b}$ & $\mathrm{c}$ \\
$\mathrm{a}$ & $\mathrm{a}$ & 0 & $\mathrm{c}$ & $\mathrm{b}$ \\
$\mathrm{b}$ & $\mathrm{b}$ & $\mathrm{c}$ & 0 & $\mathrm{a}$ \\
$\mathrm{c}$ & $\mathrm{c}$ & $\mathrm{b}$ & $\mathrm{a}$ & 0
\end{tabular}

\begin{tabular}{l|llll}
$\cdot$ & 0 & $\mathrm{a}$ & $\mathrm{b}$ & $\mathrm{c}$ \\
\hline 0 & 0 & 0 & 0 & 0 \\
$\mathrm{a}$ & 0 & 0 & $\mathrm{a}$ & $\mathrm{a}$ \\
$\mathrm{b}$ & 0 & 0 & $\mathrm{~b}$ & $\mathrm{~b}$ \\
$\mathrm{c}$ & 0 & 0 & $\mathrm{c}$ & $\mathrm{c}$
\end{tabular}

Then $(X ; *, \cdot, 0)$ is a BF-semigroup. Now, $\mu$ is a fuzzy sub BF-semigroup of $X$, where $\mu(0)=0.7=\mu(a)$ and $\mu(\mathrm{b})=0.4=\mu(\mathrm{c})$.

Lemma 4.3. Let $\mu$ be a fuzzy sub BF-semigroup of $X$. Then $\mu(0) \geqslant \mu(x)$ for all $x \in X$. Moreover, if $\mu$ is onto, then $\mu(0)=1$.

Proof. The first statement follows from ([1, Lemma 3.3]). Now, if $\mu$ is onto, then there exists an $x \in X$ such that $\mu(x)=1$. Therefore, $1=\mu(x) \leqslant \mu(0) \leqslant 1$, that is, $\mu(0)=1$.

Theorem 4.4. Let $\mu$ be a fuzzy sub BF-semigroup of $X$. Then there exists a sequence $\left\langle x_{n}\right\rangle$ in $X$ such that $\lim _{n \rightarrow \infty} \mu\left(x_{n}\right)=1$ if and only if $\mu(0)=1$.

Proof. The first part of the theorem follows from ([1, Theorem 3.5]). Suppose that $\mu(0)=1$. Consider the sequence $\left\langle x_{n}\right\rangle=\langle 0,0, \cdots\rangle$ in $X$. Then clearly $\lim _{n \rightarrow \infty} \mu\left(x_{n}\right)=1$.

Proposition 4.5. Let $\mu$ be a fuzzy sub BF-semigroup of $X$. Then the set $X_{\mu}=\{x \in X: \mu(x)=\mu(0)\}$ is a sub BF-semigroup of $\mathrm{X}$.

Proof. Let $x, y \in X_{\mu}$. Then $\mu(x)=\mu(0)=\mu(y)$. From ([1, Theorem 3.14]), $x * y \in X_{\mu}$. Now,

$$
\mu(x \cdot y) \geqslant \min \{\mu(x), \mu(y)\}=\mu(0) .
$$

Applying Lemma 4.3, $\mu(x \cdot y)=\mu(0)$. Hence, $x \cdot y \in X_{\mu}$. Therefore, $X_{\mu}$ is a sub BF-semigroup of $X$.

Lemma 4.6. If $\mu$ is a fuzzy sub BF-semigroup of $\mathrm{X}$, then

(a) $\mu(0 * x)=\mu(x)$;

(b) $\mu(x *(0 * y)) \geqslant \min \{\mu(x), \mu(y)\} ;$

(c) $\mu(x * y)=\mu(y * x)$.

Proof.

(a): By Lemma 4.3, $\mu(0 * x) \geqslant \min \{\mu(0), \mu(x)\}=\mu(x)$. By Proposition 2.7 (a) and Lemma 4.3, $\mu(x)=$ $\mu(0 *(0 * x)) \geqslant \min \{\mu(0), \mu(0 * x)\}=\mu(0 * x)$. Therefore, $\mu(0 * x)=\mu(x)$.

(b): By (a), $\mu(x *(0 * y)) \geqslant \min \{\mu(x), \mu(0 * y)\}=\min \{\mu(x), \mu(y)\}$.

(c): By (BF) and (a), $\mu(x * y)=\mu(0 *(y * x))=\mu(y * x)$.

Theorem 4.7. Suppose that $\mu$ is a fuzzy sub BF-semigroup of a $\mathrm{BF}_{1}$-semigroup $\mathrm{X}$. If $\mu(\mathrm{x} * \mathrm{y})=\mu(0)$, then $\mu(x)=\mu(y)$.

Proof. Let $x, y \in X$ and $\mu(x * y)=\mu(0)$. By $(B G), x=(x * y) *(0 * y)$. Thus, by Lemma 4.6 (a) and Lemma 4.3, we have $\mu(x)=\mu((x * y) *(0 * y)) \geqslant \min \{\mu(x * y), \mu(0 * y)\}=\min \{\mu(0), \mu(y)\}=\mu(y)$. By Lemma 4.6 (c), $\mu(0)=\mu(x * y)=\mu(y * x)$. Applying similar argument as above, we obtain $\mu(y) \geqslant \mu(x)$. Therefore, $\mu(x)=\mu(y)$.

Theorem 4.7 does not hold for BF-semigroup as shown in the following example.

Example 4.8. Consider the BF-semigroup $(X ; *, \cdot, 0)$ in Example 2.5. Now, $\mu$ is a fuzzy sub BF-semigroup of $X$, where $\mu(0)=0.5=\mu(a)$ and $\mu(b)=0.2$. Moreover, $\mu(a * b)=\mu(0)$ but $\mu(a)=0.5 \neq 0.2=\mu(b)$. 
The upper level set $U(\mu ; t)$ is the set $U(\mu ; t)=\{x \in X: \mu(x) \geqslant t\}$, where $0 \leqslant t \leqslant 1$.

Theorem 4.9. Suppose that $\mathrm{U}(\mu ; \mathrm{t})$ is nonempty. Then $\mu$ is a fuzzy sub BF-semigroup of $\mathrm{X}$ if and only if $\mathrm{U}(\mu ; \mathrm{t})$ is a sub BF-semigroup of $\mathrm{X}$.

Proof. The "only if" statement is clear. Suppose that $\mathrm{U}(\mu ; \mathrm{t})$ is a sub BF-semigroup of $\mathrm{X}$. If there are $x_{0}, y_{0} \in X$ such that $\mu\left(x_{0} * y_{0}\right)<\min \left\{\mu\left(x_{0}\right), \mu\left(y_{0}\right)\right\}$, then taking $t_{0}=\frac{1}{2}\left(\mu\left(x_{0} * y_{0}\right)+\min \left\{\mu\left(x_{0}\right), \mu\left(y_{0}\right)\right\}\right)$, $\mu\left(x_{0} * y_{0}\right)<t_{0}<\min \left\{\mu\left(x_{0}\right), \mu\left(y_{0}\right)\right\}$. Hence, $x_{0}, y_{0} \in U\left(\mu ; t_{0}\right)$, but $x_{0} * y_{0} \notin U\left(\mu ; t_{0}\right)$, a contradiction. This means that $\mu(x * y) \geqslant \min \{\mu(x), \mu(y)\}$ for all $x, y \in X$. Similarly, $\mu(x \cdot y) \geqslant \min \{\mu(x), \mu(y)\}$ for all $x, y \in X$. Therefore, $\mu$ is a fuzzy sub BF-semigroup of $X$.

Theorem 4.10. Let $\mathrm{N}$ be a nonempty subset of a BF-semigroup $\mathrm{X}$. Suppose that $\mu_{\mathrm{N}}$ is a fuzzy set in $\mathrm{X}$ defined by

$$
\mu_{N}(x)= \begin{cases}\alpha, & x \in N, \\ \beta, & \text { otherwise, }\end{cases}
$$

for all $x \in X$ and $\alpha, \beta \in[0,1]$ with $\alpha>\beta$. Then $\mu_{N}$ is a fuzzy sub BF-semigroup of $X$ if and only if $N$ is a sub BF-semigroup of $\mathrm{X}$. Moreover, in this case, $\mathrm{X}_{\mu_{\mathrm{N}}}=\mathrm{N}$.

Proof. Straightforward.

Corollary 4.11. A subset $\mathrm{N}$ of $\mathrm{X}$ is a sub BF-semigroup $\mathrm{X}$ if and only if $\chi_{\mathrm{N}}$ is a fuzzy sub BF-semigroup of $\mathrm{X}$.

Proof. Take $\alpha=1$ and $\beta=0$ in Theorem 4.10.

Corollary 4.12. If $\mathrm{N}$ is a sub BF-semigroup of $\mathrm{X}$, then there exists a fuzzy sub BF-semigroup $\mu$ of $\mathrm{X}$ such that $\mathrm{U}(\mu ; \mathrm{t})=\mathrm{N}$ for any $0<\mathrm{t}<1$.

Let $X$ and $Y$ be two nonempty sets and $f: X \rightarrow Y$ a mapping. Let $\mu$ a fuzzy set of $Y$. The preimage of $\mu$ under $f$, denoted by $\mu^{f}$, is the fuzzy set of $X$ defined by $\mu^{f}(x)=\mu(f(x))$ for all $x \in X$, that is, $\mu^{f}=\mu \circ f$. Let $\mu$ be a fuzzy set of $X$. The mapping $f(\mu): Y \rightarrow[0,1]$ defined by

$$
f(\mu)(y)= \begin{cases}\sup _{x \in f^{-1}(y)}\{\mu(x)\}, & f^{-1}(y) \neq \varnothing, \\ 0, & f^{-1}(y)=\varnothing,\end{cases}
$$

is called the image of $\mu$ under $f$, where $f^{-1}(y)=\{x \in X: f(x)=y\}$.

Let $f: X \rightarrow Y$ be a BFs-homomorphism. If $\mu$ is a fuzzy sub BF-semigroup, then $f(\mu)(0)=\mu(0)$ and $\mu^{f}(0)=\mu(0)$. Let $N$ be a nonempty subset of a BF-semigroup $X$. A fuzzy set $\mu$ is said to have the supremum property if there exists $n_{0} \in N$ such that $\mu\left(n_{0}\right)=\sup _{n \in N}\{\mu(n)\}$. If $X$ is a finite set, then $\mu: X \rightarrow[0,1]$ has the supremum property. The following theorem shows that the homomorphic image $f(\mu)$ of a fuzzy sub BF-semigroup $\mu$ is a fuzzy sub BF-semigroup of the image of $f$.

Theorem 4.13. Let $\mathrm{f}: \mathrm{X} \rightarrow \mathrm{Y}$ be a BFs-epimorphism. If $\mu$ is a fuzzy sub BF-semigroup of $\mathrm{X}$ with the supremum property, then $\mathrm{f}(\mu)$ is a fuzzy sub BF-semigroup of $\mathrm{Y}$.

Proof. Let $\mu$ be a fuzzy sub BF-semigroup of $X$ with the supremum property. Suppose that $y_{1}, y_{2} \in Y$. Since $f$ is onto, $f^{-1}\left(y_{1}\right)$ and $f^{-1}\left(y_{2}\right)$ are nonempty subsets of $X$. Since $\mu$ has the supremum property, there exist $x_{1} \in f^{-1}\left(y_{1}\right)$ and $x_{2} \in f^{-1}\left(y_{2}\right)$ such that $\mu\left(x_{1}\right)=\sup _{x \in f^{-1}\left(y_{1}\right)} \mu(x)$ and $\mu\left(x_{2}\right)=\sup _{x \in f^{-1}\left(y_{2}\right)} \mu(x)$. Since $f\left(x_{1} * x_{2}\right)=f\left(x_{1}\right) * f\left(x_{2}\right)=y_{1} * y_{2}, x_{1} * x_{2} \in f^{-1}\left(y_{1} * y_{2}\right)$. Hence,

$$
\begin{aligned}
f(\mu)\left(y_{1} * y_{2}\right) & =\sup _{x \in f^{-1}\left(y_{1} * y_{2}\right)} \mu(x) \\
& \geqslant \mu\left(x_{1} * x_{2}\right)
\end{aligned}
$$




$$
\begin{aligned}
& \geqslant \min \left\{\mu\left(x_{1}\right), \mu\left(x_{2}\right)\right\} \\
& =\min \left\{\sup _{x \in f^{-1}\left(y_{1}\right)} \mu(x), \sup _{x \in f^{-1}\left(y_{2}\right)} \mu(x)\right\} \\
& =\min \left\{f(\mu)\left(y_{1}\right), f(\mu)\left(y_{2}\right)\right\} .
\end{aligned}
$$

Similarly, $f(\mu)\left(y_{1} \cdot y_{2}\right) \geqslant \min \left\{f(\mu)\left(y_{1}\right), f(\mu)\left(y_{2}\right)\right\}$. Therefore, $f(\mu)$ is a fuzzy sub BF-semigroup of $Y$.

If $X$ is finite, then $\mu$ has the supremum property.

Corollary 4.14. If $X$ is finite, $f: X \rightarrow Y$ is a BFs-epimorphism and $\mu$ is a fuzzy sub BF-semigroup of $X$, then $f(\mu)$ is a fuzzy sub BF-semigroup of $\mathrm{Y}$.

Let $f: X \rightarrow Y$ be a BFs-homomorphism. The following theorem shows that the homomorphic preimage $\mu^{f}$ of a fuzzy sub BF-semigroup $\mu$ of $Y$ is a fuzzy sub BF-semigroup of $X$.

Theorem 4.15. Let $\mathrm{f}: \mathrm{X} \rightarrow \mathrm{Y}$ be a BFs-homomorphism. If $\mu$ is a fuzzy sub BF-semigroup of $\mathrm{Y}$, then $\mu^{\mathrm{f}}$ is a fuzzy sub BF-semigroup of $\mathrm{X}$. If $\mathrm{f}$ is onto, then the converse holds.

Proof. Let $\mu$ is a fuzzy sub BF-semigroup of $Y$. Suppose that $x, y \in X$ such that $f(x)=a$ and $f(y)=b$. Then

$$
\begin{aligned}
\mu^{f}(x * y) & =\mu(f(x * y)) \\
& =\mu(f(x) * f(y)) \\
& =\mu(a * b) \\
& \geqslant \min \{\mu(a), \mu(b)\} \\
& =\min \{\mu(f(x)), \mu(f(y))\} \\
& =\min \left\{\mu^{f}(x), \mu^{f}(y)\right\} .
\end{aligned}
$$

Similarly, $\mu^{f}(x \cdot y) \geqslant \min \left\{\mu^{f}(x), \mu^{f}(y)\right\}$. Hence, $\mu^{f}$ is a fuzzy sub BF-semigroup of $X$. For the converse, suppose that $f$ is onto and $\mu^{f}$ is a fuzzy sub BF-semigroup of $X$. Let $a, b \in Y$. Since $f$ is onto, $a=f(x)$ and $b=f(y)$ for some $x, y \in X$. Hence,

$$
\begin{aligned}
\mu(a * b) & =\mu(f(x) * f(y)) \\
& =\mu(f(x * y)) \\
& =\mu^{f}(x * y) \\
& \geqslant \min \left\{\mu^{f}(x), \mu^{f}(y)\right\} \\
& =\min \{\mu(f(x)), \mu(f(y))\} \\
& =\min \{\mu(a), \mu(b)\} .
\end{aligned}
$$

Similarly, $\mu(a \cdot b) \geqslant \min \{\mu(a), \mu(b)\}$. Therefore, $\mu$ is a fuzzy sub BF-semigroup of $Y$.

\section{Acknowledgment}

The authors would like to thank the referees for the remarks, comments, and suggestions which were incorporated into this revised version.

\section{References}

[1] A. Borumand Saeid, M. A. Rezvani, On Fuzzy BF-algebras, Int. Math. Forum, 4 (2009), 13-25. 1, 4, 4, 4

[2] J. C. Endam, J. P. Vilela, Homomorphism of BF-algebras, Math. Slovaca, 64 (2014), 13-20. 3, 3, 3, 3, 3, 3

[3] A. R. Hadipour, Generalized Fuzzy BF-algebras, Sci. Magna, 5 (2009), 39-53. 1 
[4] Y. B. Jun, E. H. Roh, H. S. Kim, On BH-algebras, Sci. Math., 1 (1998), 347-354. 1

[5] C. B. Kim, H. S. Kim, On BG-algebras, Demonstratio Math., 41 (2008), 497-505. 1

[6] K. H. Lee, First Course on Fuzzy Theory and Applications, Springer-Verlag, Berlin, (2005).

[7] A. Walendziak, On BF-algebras, Math. Slovaca, 57 (2007), 119-128. 1, 2, 2.7, 3, 3, 3 , 3

[8] L. A. Zadeh, Fuzzy sets, Inf. Control, 8 (1965), 338-353. 1 